\title{
Hydrodynamic Forces on a Rough Wall
}

\author{
Hans Albert Einstein and El-Sayed Ahmed El-Samni \\ Berkeley, California
}

The dynamic forces which a turbulent flow exerts on the individual protrusions of a rough wall have been measured. It was found that even in the case of an extremely high relative roughness, the drag force on the protrusions may be determined from the logarithmic friction laws. A very significant lift force which tends to pull the protrusions away from the wall and into the flow was measured. This lift force was divided into two parts: A constant average value and a random fluctuation superimposed over the average. A careful statistical analysis showed that the frequency of different pressures or lift forces at the wall follows very accurately the normal error law. This fact seems to indicate that in the description of turbulence near a rough wall, the pressures must be regarded as the primary influence, not the velocities.

\section{THE PROBLEM}

$\mathrm{T}$ HE study described in this paper originally was initiated with the primary purpose of studying the dynamic forces acting on particles in the surface layer of a sediment bed. The statistical distribution of these forces should permit the prediction of the frequency with which solid particles are removed from a stream bed by a given flow. It has been shown that the sediment motion on such a bed may be determined from the above-mentioned frequency with which the lift forces overcome the particle weight in conjunction with an independent statistical description of the subsequent particle motion. ${ }^{1}$

This paper deals only with the forces which the flow exerts on any such particles in the surface layer of the bed over which a turbulent fluid flows. Basically, it should be possible to derive these forces from the turbulent flow patterns near a rough wall. But the turbulence at such a wall has never been studied systematically, either theoretically or experimentally. It was mandatory, therefore, to obtain first some basic experimental data.

Fundamentally, two different approaches can be used: One is to investigate the flow pattern around the protrusions of the wall and to describe it by means of the statistical turbulence theory. The forces acting on the individual particles or roughness protrusions of the boundary must then be calculated from the flow field. A second, more direct, but also more empirical approach is the direct measurement of the forces. This second approach was chosen because it was believed to be the quicker and the more reliable of the two.

The only existing theory of the turbulent flow pattern along a rough wall is that based on Karman's similarity theorem. The resulting equation for the distribution of the average velocity as a function of the distance from the wall is

$$
u / u_{*}^{\prime}=C_{1}+5.75 \log _{10}\left(y / K_{s}\right) .
$$

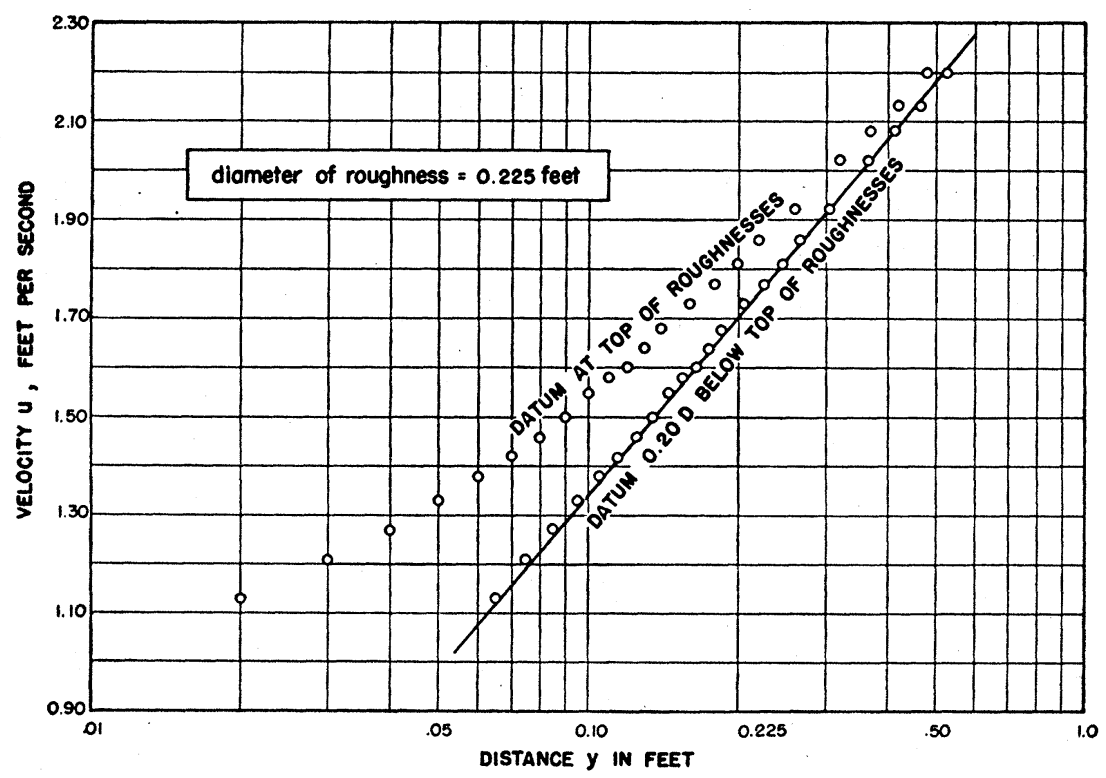

FIG 1. Vertical velocity distribution at center section.

${ }^{1}$ H. A. Einstein, "Der Geschiebetrieb als Wahrscheinlichkeits-Problem," Verl. Gebr. Leemann (Zürich, 1936). 


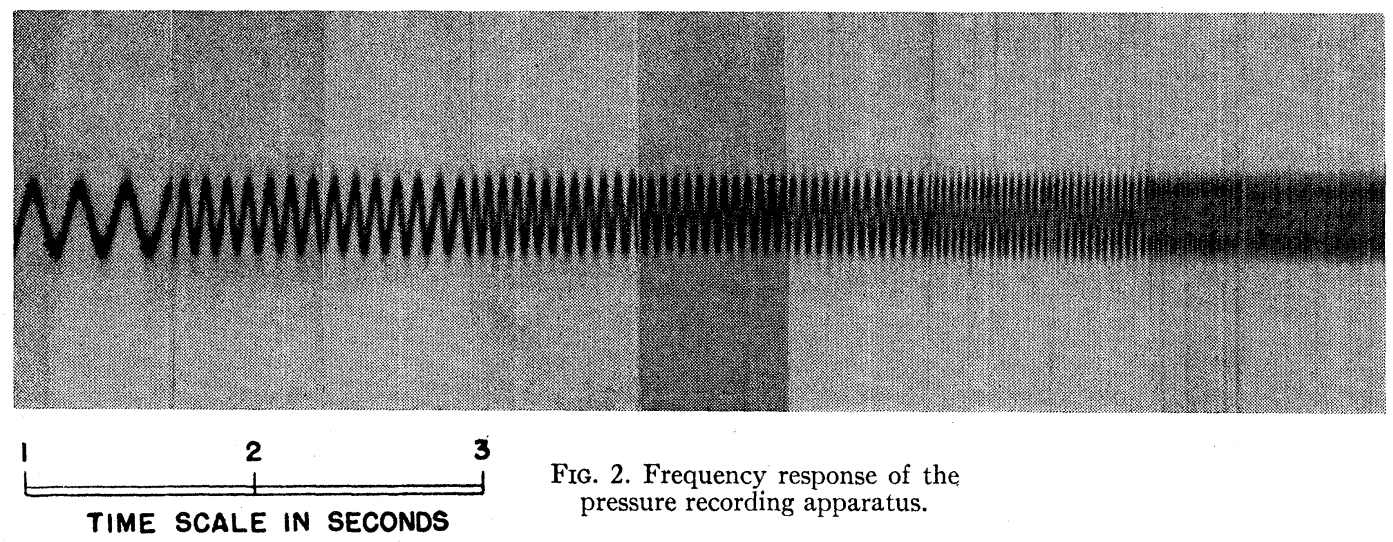

The corresponding friction formula

$$
\bar{u} / u_{*}=C_{2}+5.75 \log _{10}\left(R / K_{s}\right)
$$

shows that the roughness $K_{s}$ is the length scale of the problem. $u$ is hereby the velocity at a distance $y$ from the wall with the roughness $K_{s}$ while $\bar{u}$ is the average velocity over the hydraulic radius $R ; u_{*}=\left(\tau_{0} / \rho\right)^{\frac{1}{2}}$ is the friction velocity, $C_{1}$ and $C_{2}$ are constants. It must be expected, therefore, that also the pressure distribution can be described in a dimensionless form, with the roughness $K_{s}$ as a linear scale. From this, it was further concluded that any convenient size particle could be used to find the desired statistical pressure distributions as long as it is sufficiently large to make the surface hydraulically rough. No upper limitation can be given for the size of the particles. With the available instruments for the static and dynamic pressure measurement, the choice of the largest possible roughness was desirable. In view of the basic character of the study, plastic spherical balls, 0.225 feet in diameter, were selected as one type of roughness. In order to prevent the seepage of water between these balls and the bottom of the flume, only the upper half of the spheres were used by gluing the hemispheres in a hexagonal pattern to the bottom of the flume. The horizontal and vertical component of the average dynamic force on this extremely rough surface then were determined separately.

\section{THE AVERAGE SHEAR}

The horizontal force or over-all shear stress was determined by two different methods: First, by measuring vertical velocity distributions with a small Pitot tube, it was possible to determine the local value of the shear stress. Figure 1 shows the semi-logarithmic plot of a characteristic velocity distribution. It may be seen from Eq. (1) that the velocity must follow a straight line if plotted against the logarithm of the distance from the wall. The slope of this line represents $u^{*}=\left(\tau_{0} / \rho\right)^{\frac{1}{2}}$, where $\tau_{0}$ is the shear stress along the bed. A large number of these distributions were measured, and it was found that irrespective of their location, they plotted as straight lines if the theoretical wall was assumed to be at a distance of $0.2 K_{8}$ below a plain tangent to the top of the spheres whose diameter is $K_{s}$. These shear stresses were integrated over the entire bed. The side wall friction was considered in the analysis by the method previously used in studies on sediment transportation. ${ }^{2}$ Within an accuracy of 2-3 percent the total horizontal shear forces was found to equal the horizontal component of the weight S.L.A.W., wherein $S$ is the slope, $L$ the length, $A$ the cross-sectional area of the flow under consideration, and $W$ the specific weight of water. The constant $C_{1}$ (Eq. 1) hereby was found to have the value 8.50 as previously determined by Keulegan ${ }^{3}$ for the smaller relative roughnesses used by Nikuradse's ${ }^{4}$ experiments.

\section{THE AVERAGE LIFT}

The average lift force on the hemispheres was measured directly as a pressure difference. The procedure in making such measurements was as follows: If a lift force is exerted on the top layer of a stream bed, the solid support of the sediment particles is relieved of part of their load and this load is transmitted hydrostatically to the fluid between the solid bed particles. Thus, it must be possible to detect and measure this lift as a general pressure increase of the pore fluid in the bed. It was measured by means of micrometer-pointgages, reading to $1 / 10,000 \mathrm{ft}$. and applied to the free water surfaces in pressure wells alternately connected to pressure taps in the flume side wall above the hemispheres and to other taps in the flume floor between the hemispheres. The pressure between hemispheres always was found to be higher than that in the side wall. In an attempt to describe this pressure difference in the form

$$
\Delta p=C_{L}(\rho / 2) u^{2},
$$

2 See H. A. Einstein "Formulas for the transport of bed load" Appendix II, paper 2140 Trans. A.S.C.E. Vol. 107 (1942).

${ }^{3}$ G. H. Keulegan, "Laws of turbulent flow in open channels" Research Paper 1151, National Bureau of Standards, Vol. 21 No. 6 (1938).

${ }^{4}$ J. Nikuradse, "Stromungsgesetze in rauhen Rohren" V.d.I. Forschnngshefr 361 (1933). 


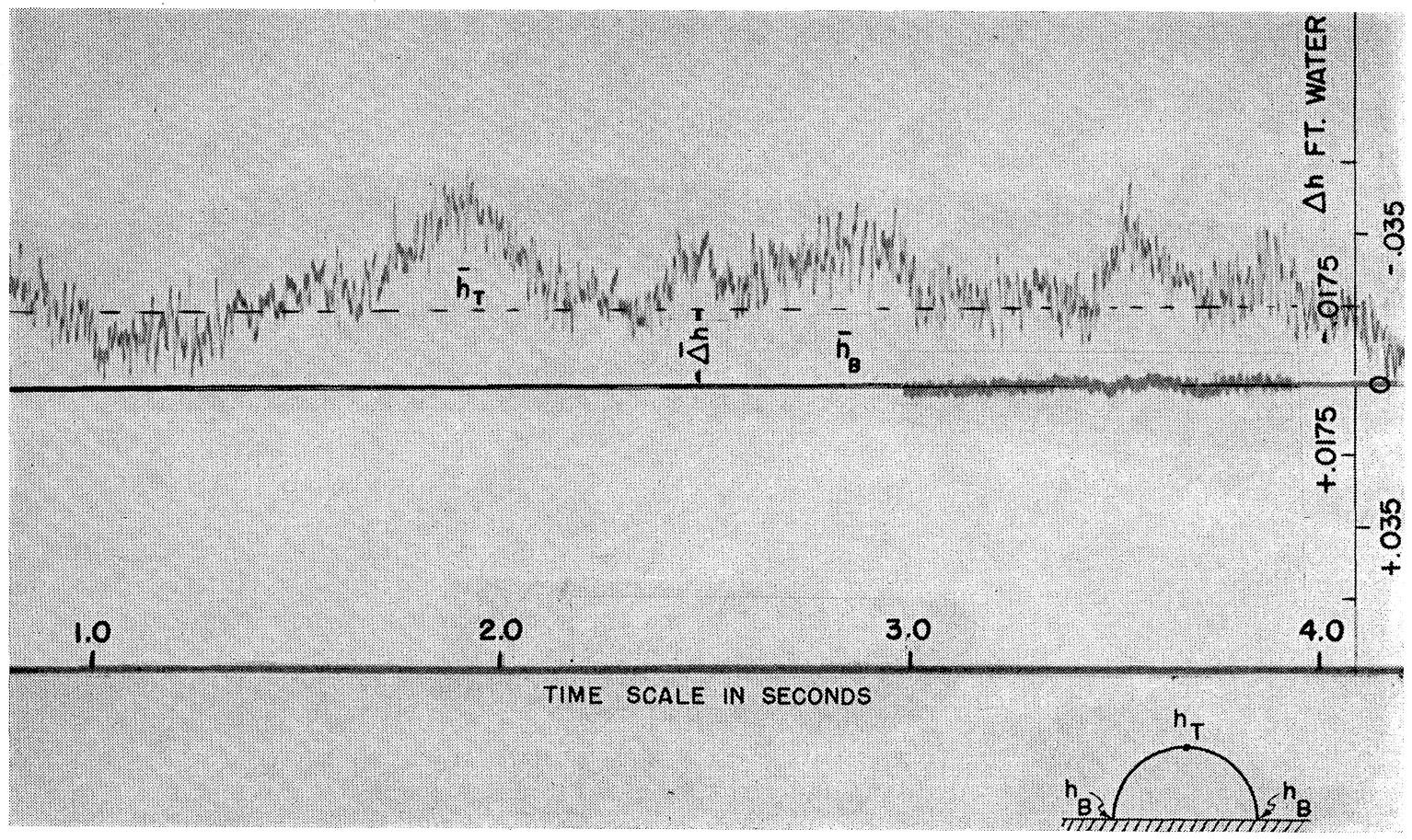

FIG. 3. Pressure record.

the lift coefficient $C_{L}$ was found to have a constant value of 0.178 if and only if the flow velocity was measured at a distance of 0.35 diameters from the theoretical wall (as formerly defined). Similarly let us anticipate that the distance at which the representative velocity exists must be measured in sphere diameters. It is very interesting to note that for every other reference distance, the lift coefficient varies with the flow condition.

\section{TURBULENT FLUCTUATIONS OF THE LIFT}

Observation of the behavior of sediment particles in motion suggests that large instantaneous variations of

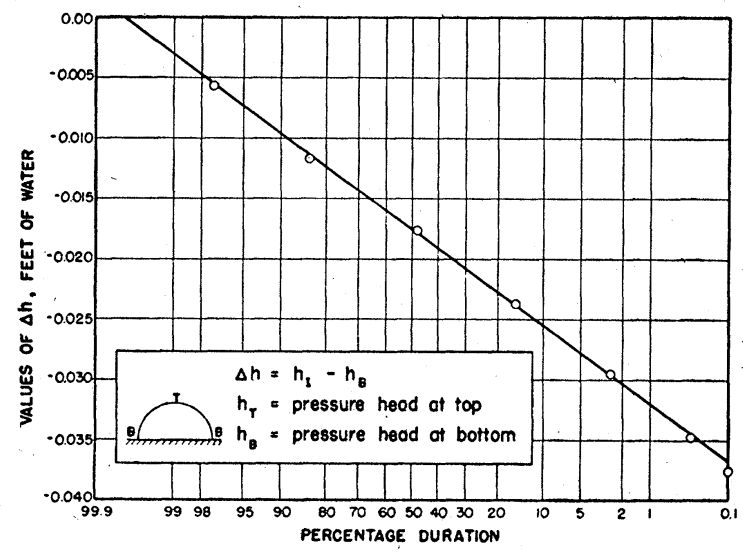

FIG. 4. Duration of lift pressures. the lift force must exist. As this variation influences very significantly the stability of a bed particle, the measurement of these fluctuations was deemed to be very important.

As a pressure recording instrument a Trimount pressure cell with AC-generator and amplifier and a Hathaway Magnetic oscillograph were available. The frequency response of the combination for frequencies up to 50 RPS was found to be perfect (see Fig. 2). Higher frequencies are unimportant for the large hemispheres at the low velocities used in these experiments.

The average lift was measured as pressure difference between the top and the bottom of the spheres. The pressure fluctuations were measured for these two pressures individually. Unfortunately, the fluctuations of the pressure difference itself could not be recorded directly for reasons of instrumentation. The fluctuations of the two pressures were measured individually, however, as seen in Fig. 3 , where $\bar{h}_{I}$ represents the long-time average of the pressure on the top of the hemisphere as recorded through a large number of holes covering the top $60^{\circ}$ segment of the sphere. The recorded fluctuations around this average are of the same order of magnitude as the difference $\langle\Delta h\rangle$ between $\bar{h}_{I}$ and the average pressure $\bar{h}_{B}$ between and below the spheres. A short record of the pressure fluctuations around $\bar{h}_{B}$ is shown in Fig. 3. This diagram may be interpreted as follows: $\bar{h}_{I}$ represents the static pressure in the free flow, $\bar{h}_{B}$ is the pressure between balls, $\langle\Delta h\rangle=0.0175 \mathrm{ft}$. of water higher than $\bar{h}_{I}$. The pressure $\bar{h}_{B}$ is something like a 
Fig. 5. Pressure fluctuations.

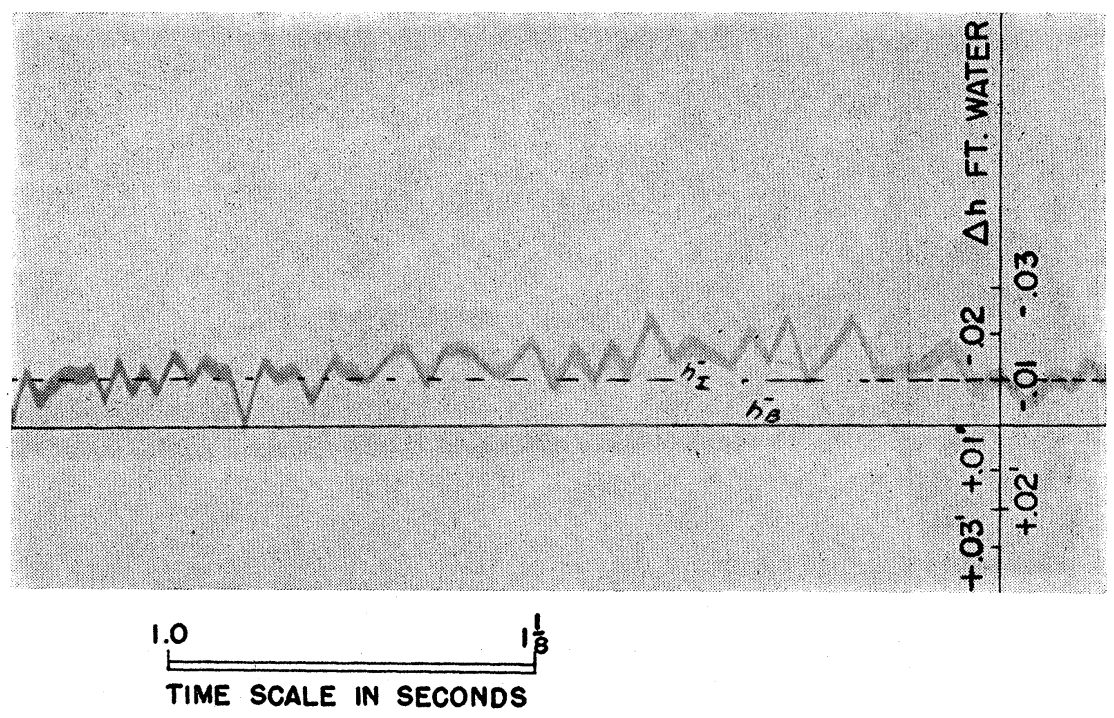

stagnation pressure because $h_{I}$ often approaches but never surpasses $\vec{h}_{B}$. The small fluctuations of the pressure $\bar{h}_{B}$ can be neglected.

In order to describe the fluctuations of $\bar{h}_{I}$ in more detail, a duration curve of pressures was constructed for several pressure records and plotted on probability paper (see Fig. 4). To the authors' astonishment, these curves all proved to be perfectly straight, proving that the pressures at this wall are statistically distributed according to the normal error law. One is tempted to conclude from this fact, that the pressures are the basic variables in the statistical description of turbulence in the vicinity of a wall, rather than the velocity. The duration curves of pressure permitted the determination of the standard deviation of pressure which was equal to $\langle\Delta h\rangle / 2.75$ with only insignificant variation between runs. It must be emphasized, however, that the duration curves were made from records of 60 and more seconds duration. In order to study the details of the fluctuations, several records were made with a larger time scale (see Fig. 5). It becomes apparent there that the fluctuations are not regular, even if a period of about $1 / 50$ second seems to be predominant. Only a statistical description of these fluctuations appears to be adequate.

\section{NATURAL GRAVEL}

The primary purpose of this study was the determination of the dynamic forces on particles of a natural sediment deposit. The hemispheres studied thus far could be considered as an idealized sediment deposit, but some measurements on actual sediment particles were necessary to verify the applicability of the results to natural sediment. This verification was restricted, however, to the measurement of average values of the shear force and of the lift.

In contrast to the hemispheres, the natural sediment is irregular in three different respects: (1) The shape of the individual particle is irregular. (2) The size and shape of the particles are different from one another. (3) The location of the different particles with respect to the theoretical wall is irregular. The influence of this irregularity of any natural sediment was tested by repetition of a part of the experiments with a natural gravel. The gravel had about the same average size as the spheres, but had a considerable spread of grain sizes as described by a sieve-size analysis. The gravel was placed in a flume, $1-\mathrm{ft}$. wide and about $30-\mathrm{ft}$. long, to a depth of 6-8-inches thick with the surface made as smooth as possible, only flows were tested which did not cause particle motion.

The first tests involved the shear determination by the measurement of velocity distributions. Again, it was observed that a plane could be found containing all points which will, as the theoretical wall points of the velocity distribution, make the distributions appear as straight lines on a semilogarithmic graph. This plane was found to be located $0.040-\mathrm{ft}$. below the top points of the gravel as determined by laying a board on the bed and by measuring the location of the lower underside of the board with the Pitot tube. If this distance was again assumed to represent 20 percent of the representative grain size, this size locating the theoretical bed is $0.200 \mathrm{ft}$. From the mechanical analysis it was found that 67 percent of the particles (by weight) are smaller than this size.

In these same experiments, the energy slope was measured and the friction along the side walls was computed. This permitted again a test to be made on the applicability of Eq. (2). Very close agreement was achieved if the roughness $K_{s}$ was chosen to equal $0.200 \mathrm{ft}$., the identical size which was found to be representative for the location of the theoretical wall. This value confirms very closely the rule of thumb previously given by the senior author. ${ }^{5}$

\footnotetext{
5 H. A. Einstein, "Flow on a movable bed" Proc. Second Hydraulics Conference, Iowa City, Bulletin 27, 1943.
} 
The average dynamic lift on the bed particles was measured by a similar method to that used in the studies with the hemispheres; that is, the difference of pressures in tap holes of the channel wall above the bed and in perforated tubes under the bed was measured by means of micro-point-gauges. The average lift pressure was found to be again

$$
\Delta p=0.178 \rho\left(u^{2} / 2\right),
$$

if the velocity $u$ again was measured 0.35 -grain diameters from the theoretical wall and the sieve size of that grain size was introduced as the diameter of which 35 percent (by weight) of the mixture was finer. This diameter is considerably different from that describing the roughness of the mixture, but equals that size which the senior author previously found to describe the mixture in an equation for the rate of bed-load load transportation. ${ }^{6}$ Since the lift has been described

${ }^{6}$ H. A. Einstein, Trans. A.S.C.E. 107, 571 (1942). as the major moving force of bed-load, ${ }^{2}$ the agreement of the representative grain diameters for lift and transport of mixtures seems to be significant.

The measurement of the fluctuations of this lift force was not attempted, as the choice of a particle for measurement would have been arbitrary and for that reason hardly sufficiently representative to prove anything.

This paper represents a resume of the results which the junior author found in an experimental study under the general guidance of the senior author and which is described in detail in a Ph.D. thesis. ${ }^{7}$ A copy of this thesis, which includes the description of the apparatus, including many photographs, tables, and graphs, all calibrations and measuring results may be made available on a loan basis to all who are especially interested.

${ }^{7}$ E. A. El Samni, "Hydrodynamic forces acting on the surface particles of a stream bed" (Ph.D. thesis, University of California, Dept. of Eng., Berkeley, 1949).

\title{
On the Transfer Rate of the Rollin-Simon Film
}

\author{
W. J. DE HAas and G. J. VAN DEN BERG \\ Kamerlingh Onnes Laboratory, Leiden University, Holland
}

$I^{\mathrm{N}}$ 1922 Kamerlingh Onnes attempted to reach the lowest possible temperature in the following manner: He surrounded a Dewar flask completely with liquid helium in such a way that heat could not penetrate by radiation nor by conduction. Inside this Dewar flask he placed still another smaller Dewar flask filled partly with liquid helium and he hoped through the lowering of the vapor pressure with molecular pumps of great speed to reach an extremely low temperature.

In this experiment there appeared a most remarkable phenomenon: With amazing rapidity liquid helium appeared outside the inner flask until the two levels of the helium in the inner and outer flask were equal. By varying this experiment he found that helium placed in the outer flask was also transferred to the inner flask. This transfer always continued until the levels in both flasks were at the same height.

The origin of this remarkable Kamerlingh Onnes effect was given by Simon and Rollin. They assumed that the wall of the flask was covered by an extremely thin film of helium and in this way the helium was "syphoned" from one place to the other. This RollinSimon film has been investigated in Oxford, Cambridge, Bristol and Russia.

Daunt and Mendelssohn carried out extensive experiments on the transport phenomena in the He II film; meanwhile Atkins in Cambridge got quite different results. The principal results which Daunt and Mendelssohn obtained were:

1. The transport velocity is a constant quantity independent of the difference in levels except for the case in which the He II in the tube is less than $1 \mathrm{~cm}$ from the top.

2. The transport velocity increases with falling temperature from the $\lambda$-point to $1.5^{\circ} \mathrm{K}$ and remains approximately constant below this temperature.

3. The transport velocity is independent of the material over which the film creeps.

The results of Atkins, however, are quite different. $\mathrm{He}$ finds the maximum transport velocity about 13 times greater than Daunt and Mendelssohn, and moreover this velocity is not constant but depends on the distance of the highest level to the top of the tube. As Atkins did not give many details of his apparatus it is difficult to reach definite conclusions concerning his results.

\section{INVESTIGATIONS AT LEIDEN}

Since the film has a very large surface area it is assumed that its thickness will be sensitive to incident radiation.

In order to avoid the effects of radiation one must reject all direct methods of visual observations. The principle which we therefore adopted as a criterion for measurement was the decrease of weight with time of a tube filled with He II from which the film creeps. 

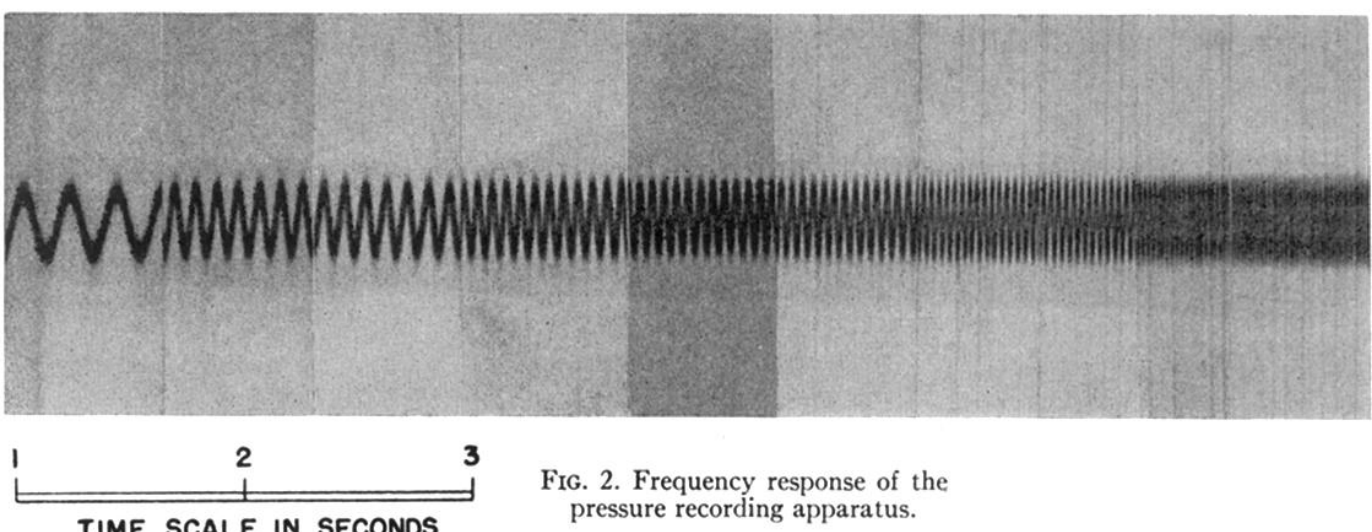

FIG. 2. Frequency response of the

TIME SCALE IN SECONDS pressure recording apparatus. 


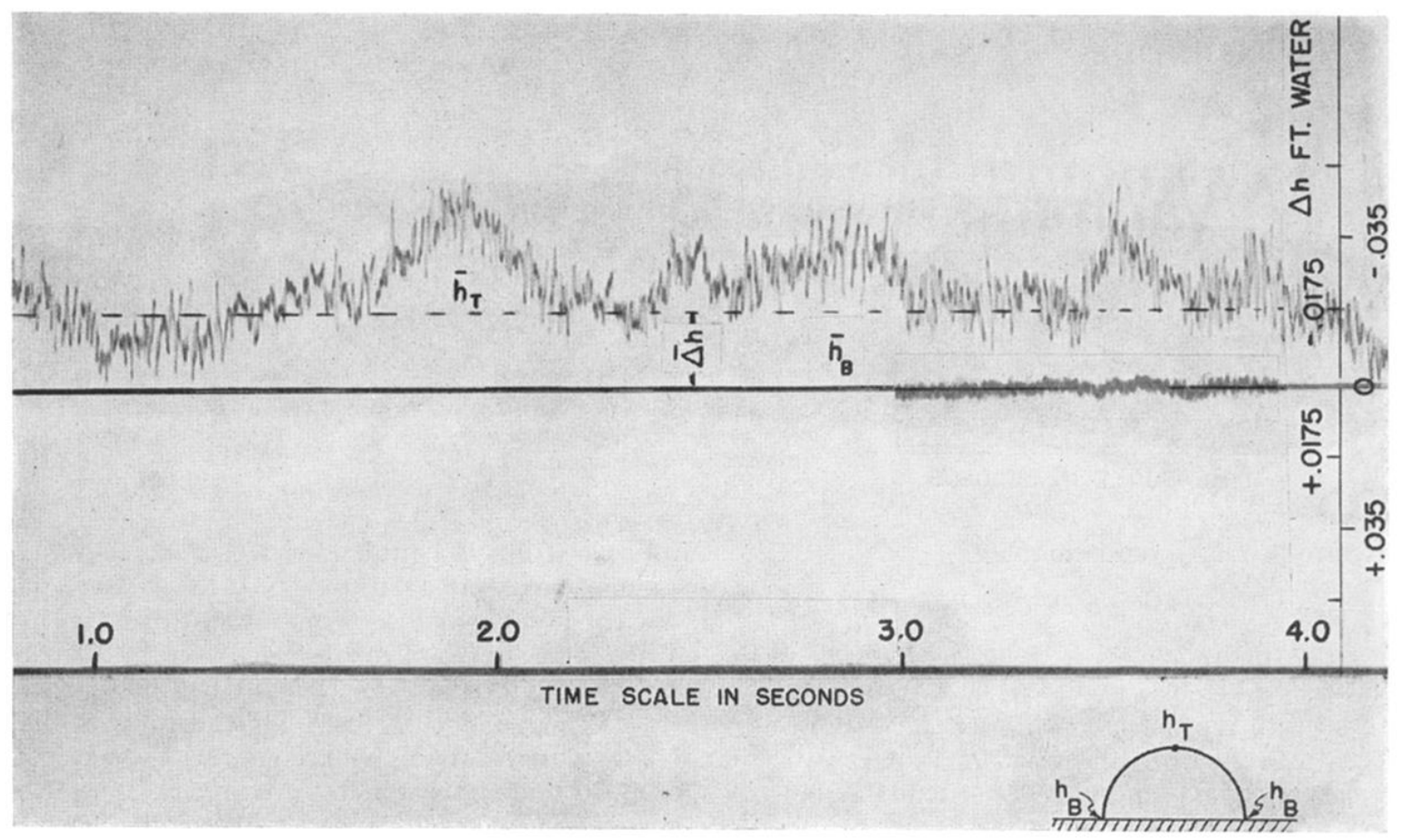

Fig. 3. Pressure record. 
FIG. 5. Pressure fluctuations.

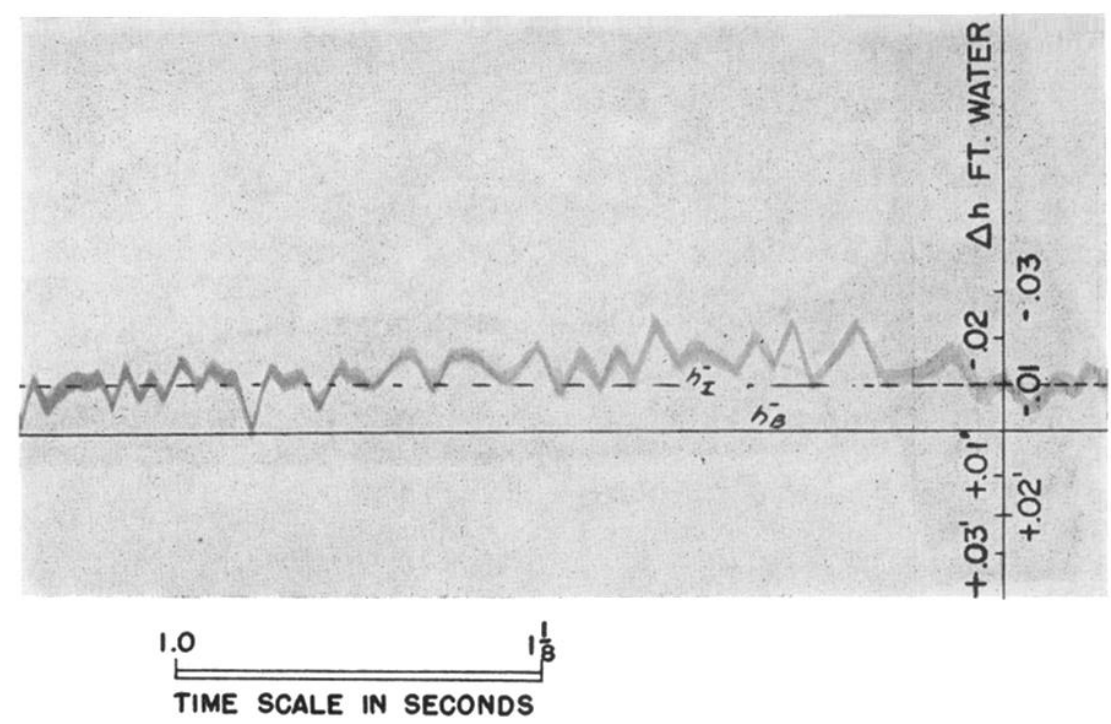

